\title{
MINIMAL INDICES AND MINIMAL BASES VIA FILTRATIONS*
}

\author{
D. STEVEN MACKEY ${ }^{\dagger}$
}

\begin{abstract}
A new way to formulate the notions of minimal basis and minimal indices is developed in this paper, based on the concept of a filtration of a vector space. The goal is to provide useful new tools for working with these important concepts, as well as to gain deeper insight into their fundamental nature. This approach also readily reveals a strong minimality property of minimal indices, from which follows a characterization of the vector polynomial bases in rational vector spaces. The effectiveness of this new formulation is further illustrated by proving several fundamental properties: the invariance of the minimal indices of a matrix polynomial under field extension, the direct sum property of minimal indices, the polynomial linear combination property, and the predictable degree property.
\end{abstract}

Key words. Singular matrix polynomial, Rational subspace, Minimal indices, Minimal basis, Filtration, Flag.

AMS subject classifications. 15A54, 15A21, 15A22.

1. Introduction. Minimal indices and bases are quantities commonly associated with singular matrix polynomials, and thus play a significant role in a number of applications, especially in systems and control theory $[7,15,19,20]$; they also are important in algebraic coding theory $[6,17,18,20]$. Although they have been defined in the literature in several different ways [7,9], these definitions have been shown to lead to the same quantities [3]. The purpose of this note is to introduce a new formulation of the notions of minimal basis and minimal indices, with the goals of:

- developing new tools for effectively working with these important concepts and

- simplifying the conceptual foundation, so as to smoothly unify the classical approaches to minimal indices and to make the well-definedness of minimal indices as transparent as possible.

This new formulation takes the algorithmic approach described in [9] by Gantmacher (and attributed to Kronecker) as a starting point, but is motivated by the following simple idea. Rather than deal with the special polynomial bases produced by Kronecker's algorithm, which are far from canonical due to the many arbitrary choices made in generating them, focus instead on the underlying subspaces from which these choices are made. These subspaces are uniquely defined, canonical objects which more clearly and directly reveal the intrinsic nature of minimal indices, and form the building blocks of the filtration at the heart of the new formulation.

Although the motivating idea is simple, some preliminary work is required to set up the appropriate definitions, terminology, and notation needed to effectively implement this idea. Section 2 begins this background work by reviewing the notion of filtration and giving some examples; Section 3 continues these preliminaries by recalling the two "classical" ways of defining minimal bases and indices. Section 3 then goes on to introduce the new characterization of minimal bases and indices by means of a special filtration called

\footnotetext{
*Received by the editors on December 27, 2019. Accepted for publication on March 13, 2021. Handling Editor: Bryan Shader.

${ }^{\dagger}$ Department of Mathematics, Western Michigan University, Kalamazoo, MI 49008, USA (steve.mackey@wmich.edu). Supported by National Science Foundation grant DMS-1016224 and by Ministerio de Economía y Competitividad of Spain through grant MTM2015-65798-P.
} 
the "degree filtration", and from this develops several new tools for proving results about minimal indices and minimal bases. Section 4 shows how the filtration point of view reveals a "strong" minimality property of minimal indices, which is then used to unify and simplify the connection between the two classical approaches to minimal indices. Finally, the effectiveness of these new tools and this new point of view is illustrated in Sections 5, 6, and 7 by proving several properties: the invariance of the minimal indices of a matrix polynomial under field extension, the behavior of minimal indices under direct sums, and the polynomial linear combination and predictable degree properties.

2. Filtrations. Let us begin with a quote from Hilton \& Wylie's classic text on algebraic topology [11, p. 395]:

We now introduce the notion of a filtration. It has a rather wide application; a construct may be said to be filtered if an increasing sequence of sub-constructs is selected which exhaust the whole construct.

Clearly all kinds of mathematical objects can be filtered: topological spaces, groups, algebras, modules, chain complexes, etc. However, in this paper we only need to consider filtrations of vector spaces, which we now formally define.

Definition 2.1 (Filtration of a Vector Space). A filtration $\mathcal{F}$ of a vector space $V$ is an infinite nested sequence of subspaces of $V$,

$$
\mathcal{F}: W_{0} \subseteq W_{1} \subseteq W_{2} \subseteq W_{3} \subseteq \cdots,
$$

such that $\bigcup_{i=0}^{\infty} W_{i}=V$. A vector space $V$ equipped with a filtration $\mathcal{F}$ as in (2.1) is said to be a filtered vector space.

For our purposes, it will be convenient to allow $W_{i}=W_{j}$ for $i \neq j$ in (2.1), although in some contexts authors require the subspaces in a filtration to be distinct. In fact, the filtrations of most interest to us will usually not have distinct subspaces. The inclusion relations in (2.1) of course imply that

$$
\operatorname{dim} W_{0} \leq \operatorname{dim} W_{1} \leq \operatorname{dim} W_{2} \leq \cdots
$$

Definition 2.2 (Dimension Sequence of a Filtration). The infinite sequence of numbers (dim $W_{0}$, $\left.\operatorname{dim} W_{1}, \operatorname{dim} W_{2}, \ldots\right)$ in $(2.2)$ will be referred to as the dimension sequence of the filtration $\mathcal{F}$, and denoted $\operatorname{dim} \mathcal{F}$.

EXAMPLE 2.3.

(a) For $V=\mathbb{F}^{n}$, the standard basis $\left\{\mathrm{e}_{1}, \mathrm{e}_{2}, \ldots, \mathrm{e}_{n}\right\}$ induces the "standard" filtration

$$
\{0\} \subseteq \operatorname{span}\left\{\mathrm{e}_{1}\right\} \subseteq \operatorname{span}\left\{\mathrm{e}_{1}, \mathrm{e}_{2}\right\} \subseteq \cdots \subseteq \operatorname{span}\left\{\mathrm{e}_{1}, \mathrm{e}_{2}, \ldots, \mathrm{e}_{n-1}\right\} \subseteq V \subseteq V \subseteq \cdots
$$

(b) Indeed any ordered list of vectors $\left\{\mathrm{v}_{1}, \mathrm{v}_{2}, \ldots, \mathrm{v}_{m}\right\}$ induces an associated filtration of the vector space $V=\operatorname{span}\left\{\mathrm{v}_{1}, \mathrm{v}_{2}, \ldots, \mathrm{v}_{m}\right\}$ as follows:

$$
\{0\} \subseteq \operatorname{span}\left\{\mathrm{v}_{1}\right\} \subseteq \operatorname{span}\left\{\mathrm{v}_{1}, \mathrm{v}_{2}\right\} \subseteq \cdots \subseteq \operatorname{span}\left\{\mathrm{v}_{1}, \mathrm{v}_{2}, \ldots, \mathrm{v}_{m-1}\right\} \subseteq V \subseteq V \subseteq \cdots .
$$

In particular, for any matrix $A \in \mathbb{F}^{m \times n}$, the ordered columns $\left\{\mathrm{a}_{1}, \mathrm{a}_{2}, \ldots, \mathrm{a}_{n}\right\}$ induce a filtration $\mathcal{F}(A)$ of the column space $V=\operatorname{Col}(A) \subseteq \mathbb{F}^{m}$ :

$$
\{0\} \subseteq \operatorname{span}\left\{\mathrm{a}_{1}\right\} \subseteq \operatorname{span}\left\{\mathrm{a}_{1}, \mathrm{a}_{2}\right\} \subseteq \cdots \subseteq \operatorname{span}\left\{\mathrm{a}_{1}, \mathrm{a}_{2}, \ldots, \mathrm{a}_{n-1}\right\} \subseteq V \subseteq V \subseteq \cdots .
$$


(c) A sequence of Krylov subspaces for $A \in \mathbb{F}^{n \times n}$,

$$
\operatorname{span}\{\mathrm{x}\} \subseteq \operatorname{span}\{\mathrm{x}, A \mathrm{x}\} \subseteq \operatorname{span}\left\{\mathrm{x}, A \mathrm{x}, A^{2} \mathrm{x}\right\} \subseteq \cdots,
$$

is certainly nested, but does not necessarily define a filtration for $V=\mathbb{F}^{n}$, since the condition $\bigcup_{i=0}^{\infty} W_{i}=\mathbb{F}^{n}$ may not be satisfied.

(d) Suppose $A \in \mathbb{F}^{n \times n}$ has eigenvalue $\lambda_{0} \in \mathbb{F}$, with the corresponding generalized eigenspace $\operatorname{ker}(A-$ $\left.\lambda_{0} I\right)^{n}=: V$. Then

$$
\{0\} \subseteq \operatorname{ker}\left(A-\lambda_{0} I\right) \subseteq \operatorname{ker}\left(A-\lambda_{0} I\right)^{2} \subseteq \cdots \subseteq \operatorname{ker}\left(A-\lambda_{0} I\right)^{n-1} \subseteq V \subseteq V \subseteq \cdots
$$

is a well-known sequence of nested subspaces associated with $V$. Indeed, the Jordan block structure of $A$ associated with $\lambda_{0}$ is determined by the dimension sequence of these subspaces [12], so it is reasonable to call (2.4) the Jordan filtration of the generalized eigenspace for $\lambda_{0}$.

(e) For a pair $(A, B) \in \mathbb{F}^{n \times n} \times \mathbb{F}^{n \times m}$ associated with a linear control system $\dot{x}=A x+B u$, the matrix $\mathcal{R}(A, B):=\left[\begin{array}{lllll}B & A B & A^{2} B & \cdots & A^{n-1} B\end{array}\right]$ is called the reachability (or sometimes the controllability) matrix of the pair $(A, B)$. The column space $V:=\operatorname{Col}(\mathcal{R}(A, B))$ can easily be shown to be the minimal $A$-invariant subspace containing $\operatorname{Col}(B)$; this $V$ is often referred to as the reachable subspace of the control system [23]. Letting $W_{j}:=\operatorname{Col}\left(\left[\begin{array}{lllll}B & A B & A^{2} B & \cdots & A^{j-1} B\end{array}\right]\right)$ for $j=1, \ldots, n$, then clearly

$$
\{0\} \subseteq W_{1} \subseteq W_{2} \subseteq \cdots \subseteq W_{n}=V \subseteq V \subseteq \cdots
$$

is a filtration of the reachable subspace $V$. Because of the close connection of the dimension sequence of (2.5) to the Brunovsky canonical form of the pair $(A, B)$ [23], Zaballa has suggested [25] that (2.5) might be called the Brunovsky filtration.

In a filtration $\mathcal{F}$, if for some index $m$ we have $W_{m}=V$ (and hence, $W_{n}=V$ for all $n \geq m$ ), then $\mathcal{F}$ is said to be a finite filtration and is sometimes written in the truncated fashion

$$
W_{0} \subseteq W_{1} \subseteq W_{2} \subseteq \cdots \subseteq W_{m}=V
$$

although we will not do so here. Note that any filtration of a finite dimensional space $V$ is necessarily a finite filtration, because the condition $\bigcup_{i=0}^{\infty} W_{i}=V$ forces $W_{m}=V$ to hold for some index $m$. A filtration such that $\operatorname{dim} W_{j}=j$ for all $0 \leq j \leq \operatorname{dim} V$ is called a complete filtration; a complete filtration of $\mathbb{F}^{n}$ is sometimes called a flag.

REMARK 2.4. Various types of flags, such as "Hessenberg flags" and "eigenflags" associated with a matrix, have been used as tools in a geometric approach to understanding the convergence behavior of the $Q R$-algorithm. See, for example, [1], [13, Appendix L7], [14], [22].

Definition 2.5 (Compatible Basis for a Filtration). Let $V$ be a finite dimensional filtered vector space, with a given filtration $\mathcal{F}$ as in (2.1). Then an ordered basis $\mathcal{B}=\left\{\mathrm{v}_{1}, \mathrm{v}_{2}, \ldots, \mathrm{v}_{n}\right\}$ for $V$ is said to be compatible with (or adapted to) the filtration $\mathcal{F}$ if for each subspace $W_{\ell}$ in $\mathcal{F}$ there is an initial segment $\left\{\mathrm{v}_{1}, \mathrm{v}_{2}, \ldots, \mathrm{v}_{j}\right\}$ of $\mathcal{B}$ (with $j \leq n$ ) that forms a basis for $W_{\ell}$. 
EXAMPLE 2.6.

(a) Suppose $A \in \mathbb{F}^{n \times n}$ is nonsingular. Then the (ordered) columns of $A$ form a basis compatible with the "standard" filtration (2.3) if and only if $A$ is upper triangular.

(b) Here is a well-known result expressed in the language of filtrations:

Suppose $A$ is nonsingular with $Q R$-decomposition $A=Q R$. Then the ordered columns of $Q$ form a basis compatible with the filtration $\mathcal{F}(A)$ induced by the ordered columns of $A$. Equivalently, one could express this result simply by saying that $\mathcal{F}(A)=\mathcal{F}(Q)$.

\section{Minimal bases and minimal indices.}

3.1. Two classical approaches. Minimal bases and indices were originally introduced by Kronecker as a means to help prove the uniqueness of what we now refer to as the Kronecker canonical form (KCF) for matrix pencils $L(\lambda)=\lambda X+Y$, where $X, Y \in \mathbb{C}^{m \times n}$. We begin by recalling the main points of this theory, summarizing the development in Gantmacher [9].

First some notation. Throughout the paper, $\mathbb{F}$ denotes an arbitrary field, $\mathbb{F}[\lambda]$ the ring of polynomials in the variable $\lambda$ with coefficients from $\mathbb{F}$, and $\mathbb{F}(\lambda)$ the field of rational functions over $\mathbb{F}$. Then the column vectors $\mathbb{F}(\lambda)^{n}$ form an $n$-dimensional vector space over the field $\mathbb{F}(\lambda)$, and the elements $\mathrm{v}(\lambda) \in \mathbb{F}[\lambda]^{n} \subset \mathbb{F}(\lambda)^{n}$ are the vector polynomials in $\mathbb{F}(\lambda)^{n}$. The degree of a vector polynomial is the maximum of the degrees of its component scalar polynomials.

For a matrix pencil $L(\lambda)=\lambda X+Y \in \mathbb{F}[\lambda]^{m \times n}$, viewed as a linear transformation $\mathbb{F}(\lambda)^{n} \rightarrow \mathbb{F}(\lambda)^{m}$, consider the right nullspace of $L(\lambda)$, i.e., consider

$$
\mathcal{N}_{r}(L):=\left\{\mathrm{w}(\lambda) \in \mathbb{F}(\lambda)^{n}: L(\lambda) \mathrm{w}(\lambda) \equiv 0\right\}
$$

Our goal is to find a basis for the subspace $\mathcal{N}_{r}(L)$ consisting solely of vector polynomials, but with the minimum possible degrees. In the Kronecker/Gantmacher development, this minimality is defined by the following "greedy" algorithm for constructing a vector polynomial basis.

Algorithm 3.1 (Kronecker/Gantmacher Construction).

- First choose any nonzero vector polynomial $\mathrm{v}_{1}(\lambda) \in \mathcal{N}_{r}(L)$ of minimal degree.

- Letting $V_{1}:=\operatorname{span}\left\{\mathrm{v}_{1}(\lambda)\right\}$, next choose any vector polynomial $\mathrm{v}_{2}(\lambda)$ of minimal degree in the complement $\mathcal{N}_{r}(L) \backslash V_{1}$, and extend to $\left\{\mathrm{v}_{1}(\lambda), \mathrm{v}_{2}(\lambda)\right\}$.

- With $V_{j}:=\operatorname{span}\left\{\mathrm{v}_{1}(\lambda), \ldots, \mathrm{v}_{j}(\lambda)\right\}$, continue in this fashion until a basis for $\mathcal{N}_{r}(L)$ is attained, always extending $\left\{\mathrm{v}_{1}(\lambda), \ldots, \mathrm{v}_{k-1}(\lambda)\right\}$ to $\left\{\mathrm{v}_{1}(\lambda), \ldots, \mathrm{v}_{k}(\lambda)\right\}$ by choosing a vector polynomial $\mathrm{v}_{k}(\lambda)$ of minimal degree in the remaining complement $\mathcal{N}_{r}(L) \backslash V_{k-1}$.

Definition 3.2 (K-minimal Basis). Any vector polynomial basis produced by Algorithm 3.1 is said to be a minimal basis for $\mathcal{N}_{r}(L)$ in the sense of Kronecker/Gantmacher, or a K-minimal basis for short. Such a basis is also commonly referred to as a right minimal basis for the pencil $L(\lambda)$.

It can be shown (see [9] for details) that the ordered list of degrees $\varepsilon_{1} \leq \varepsilon_{2} \leq \cdots \leq \varepsilon_{p}$, where $\varepsilon_{j}=$ $\operatorname{deg} \mathrm{v}_{j}(\lambda)$, is the same for every K-minimal basis of $\mathcal{N}_{r}(L)$, and thus displays an intrinsic feature of $\mathcal{N}_{r}(L)$.

Definition 3.3 (K-minimal Indices). The numbers $\varepsilon_{1} \leq \varepsilon_{2} \leq \cdots \leq \varepsilon_{p}$ are the K-minimal indices of $\mathcal{N}_{r}(L)$, often referred to as the right minimal indices of the pencil $L(\lambda)$. 
Clearly one can proceed analogously with the left nullspace of $L(\lambda)$, i.e.,

$$
\mathcal{N}_{\ell}(L):=\left\{\mathrm{y}(\lambda) \in \mathbb{F}(\lambda)^{m}: \mathrm{y}(\lambda)^{T} L(\lambda) \equiv 0\right\}
$$

and thus obtain left minimal bases for $L(\lambda)$, and thence the left minimal indices of $L(\lambda)$. It can be shown (again, see [9] for details) that these left and right minimal indices encode the sizes of the "singular blocks" in the KCF of $L(\lambda)$, thus proving that the "singular part" of the KCF is uniquely determined.

An examination of Algorithm 3.1 shows that $L(\lambda)$ being a matrix pencil plays no role in the discussion; it might just as well be any matrix polynomial $P(\lambda) \in \mathbb{F}[\lambda]^{m \times n}$. Thus the same development applied to the right and left nullspaces $\mathcal{N}_{r}(P) \subseteq \mathbb{F}(\lambda)^{n}$ and $\mathcal{N}_{\ell}(P) \subseteq \mathbb{F}(\lambda)^{m}$ yields right and left minimal bases for $P$, as well as right and left minimal indices for $P$. Indeed we can go one step further, and observe that even the matrix polynomial $P$ is not essential. One can apply the "greedy" algorithm to any rational subspace $V \subseteq \mathbb{F}(\lambda)^{n}$ (i.e., to an arbitrary $\mathbb{F}(\lambda)$-subspace of $\left.\mathbb{F}(\lambda)^{n}\right)$, and thus obtain K-minimal bases and K-minimal indices for any such $V$. Note that K-minimal indices are sometimes referred to in the literature as Kronecker indices $[10,24]$.

The recognition that the notions of minimal bases and indices apply to any subspace $V \subseteq \mathbb{F}(\lambda)^{n}$ is made explicit by Forney in [7], where he uses a somewhat different minimality principle to define minimal bases and minimal indices. Instead of building up vector polynomial bases one step at a time and invoking a "local" minimality condition at each step of the construction, Forney works more "globally" by assigning a single number to each vector polynomial basis; the order of a vector polynomial basis $\mathcal{B}=\left\{\mathrm{v}_{1}(\lambda), \ldots, \mathrm{v}_{p}(\lambda)\right\}$ for a subspace $V \subseteq \mathbb{F}(\lambda)^{n}$ is

$$
\operatorname{ord}(\mathcal{B}):=\sum_{i=1}^{p} \operatorname{deg} \mathrm{v}_{i}(\lambda)
$$

Thus we have the following definition.

Definition 3.4 (F-minimal Basis). A minimal basis for a subspace $V \subseteq \mathbb{F}(\lambda)^{n}$ in the sense of Forney, or an F-minimal basis, is any vector polynomial basis for $V$ with minimum order among all vector polynomial bases for $V$.

Forney then shows in [7] that the ordered degree sequence $0 \leq f_{1} \leq f_{2} \leq \cdots \leq f_{p}$, where $f_{i}=\operatorname{deg} v_{i}(\lambda)$, is the same for any F-minimal basis for $V$, thus uniquely defining the F-minimal indices for $V$.

REMARK 3.5. Note that Forney does not use the phrase F-minimal indices in [7] for the ordered degree sequence $f_{1} \leq f_{2} \leq \cdots \leq f_{p}$; instead he calls them the "invariant dynamical indices" of the subspace $V \subseteq \mathbb{F}(\lambda)^{n}$. Several later authors have referred to these as the Forney indices of $V[17,18,20]$.

It is natural to wonder whether there is any simple relationship between K-minimality and F-minimality, for either bases or indices. This question was addressed in [3].

TheOrem 3.6 (K-minimality vs. F-minimality [3, Lemma 2.4]). Consider any subspace $V \subseteq \mathbb{F}(\lambda)^{n}$. Then a vector polynomial basis $\mathcal{B}$ for $V$ is $K$-minimal if and only if it is F-minimal. Thus, the K-minimal indices are identical to the F-minimal indices for $V$.

As a consequence, we see that the minimality principles used by Kronecker/Gantmacher and Forney are equivalent, and can be used interchangeably, depending on convenience. 
3.2. Minimal bases from filtrations. We aim to reformulate the Kronecker/Gantmacher approach to minimal bases in such a way that it becomes completely transparent why all the arbitrary choices made in building a K-minimal basis always result in the same list of degrees, thereby producing a well-defined list of K-minimal indices. For an arbitrary rational subspace $V \subseteq \mathbb{F}(\lambda)^{n}$, we will see that there is an intrinsic, uniquely defined filtration induced by the notion of the degree of vector polynomials in $V$. This will be referred to as the (canonical) degree filtration of $V$, and denoted by $\mathcal{F}_{\mathrm{deg}}(V)$. It is from this particular filtration that the K-minimal bases and K-minimal indices of $V$ can be immediately recovered.

Here is how to define $\mathcal{F}_{\text {deg }}(V)$. Consider subsets of $V$ consisting of vector polynomials of bounded degree; in particular, for each integer $d \geq 0$ define

$$
\mathcal{P}_{d}(V):=\{\mathrm{v}(\lambda) \in V: \mathrm{v}(\lambda) \text { is a vector polynomial with } \operatorname{deg} \mathrm{v}(\lambda) \leq d\} .
$$

Clearly $\mathcal{P}_{d}(V)$ is an $\mathbb{F}$-subspace of $V$, but not an $\mathbb{F}(\lambda)$-subspace. Observe also that the inclusions

$$
\mathcal{P}_{0}(V) \subseteq \mathcal{P}_{1}(V) \subseteq \mathcal{P}_{2}(V) \subseteq \cdots,
$$

clearly hold. Now to get $\mathbb{F}(\lambda)$-subspaces of $V$, and thence a filtration of $V$, we simply take the $\mathbb{F}(\lambda)$-spans of these vector polynomial subsets,

$$
\mathcal{S}_{d}(V):=\operatorname{span}_{\mathbb{F}(\lambda)} \mathcal{P}_{d}(V)
$$

Note that if $\mathcal{P}_{d}(V)$ is nontrivial, then $\mathcal{S}_{d}(V)$ contains vector polynomials of unbounded degree, not just of degree at most $d$. This is because for any $\mathrm{v}(\lambda) \in \mathcal{P}_{d}(V)$, multiplying by the scalars $\lambda^{m} \in \mathbb{F}(\lambda)$ gives vector polynomials $\lambda^{m} \mathrm{v}(\lambda)$ that are in $\mathcal{S}_{d}(V)$ for every $m \in \mathbb{N}$. However, since $\mathcal{P}_{d}(V)$ is by definition a spanning set for $\mathcal{S}_{d}(V)$, we know there is always a basis of $\mathcal{S}_{d}(V)$ consisting solely of elements chosen from $\mathcal{P}_{d}(V)$; let us call any such basis a " $\mathcal{P}_{d}(V)$-basis" for $\mathcal{S}_{d}(V)$. Furthermore, any $\mathcal{P}_{d}(V)$-basis for $\mathcal{S}_{d}(V)$ can be extended to a $\mathcal{P}_{d+1}(V)$-basis for $\mathcal{S}_{d+1}(V)$. Note also that

$$
\operatorname{dim}_{\mathbb{F}(\lambda)} \mathcal{S}_{d}(V) \leq \operatorname{dim}_{\mathbb{F}} \mathcal{P}_{d}(V)
$$

i.e., the dimension of $\mathcal{S}_{d}(V)$ as an $\mathbb{F}(\lambda)$-vector space is never greater than the dimension of $\mathcal{P}_{d}(V)$ as an $\mathbb{F}$-vector space, and is often very much less. This is because any $\mathbb{F}$-basis for $\mathcal{P}_{d}(V)$ is also an $\mathbb{F}(\lambda)$-spanning set for $\mathcal{S}_{d}(V)$.

It is not hard to see that the condition $\bigcup_{d=0}^{\infty} \mathcal{S}_{d}(V)=V$ is satisfied. Suppose that $\mathrm{v}(\lambda)$ is an arbitrary element of $V$. Then there is some (scalar) polynomial $q(\lambda)$ such that $\mathrm{w}(\lambda)=q(\lambda) \mathrm{v}(\lambda)$ is a vector polynomial in $V$ (just clear all the denominators of the entries of $\mathrm{v}(\lambda)$ ), with some degree $d=\operatorname{deg} \mathrm{w}(\lambda)$. Then $\mathrm{w}(\lambda) \in \mathcal{P}_{d}(V)$, and so $\mathrm{v}(\lambda)=\left(\frac{1}{q(\lambda)}\right) \cdot \mathrm{w}(\lambda)$ is in $\mathcal{S}_{d}(V)$. Thus every element of $V$ is contained in some $\mathcal{S}_{d}(V)$, and so the equality $\bigcup_{d=0}^{\infty} \mathcal{S}_{d}(V)=V$ follows. Consequently the subspaces $\mathcal{S}_{d}(V)$ define a filtration of $V$.

Definition 3.7 (The Degree Filtration). The nested sequence

$$
\mathcal{S}_{0}(V) \subseteq \mathcal{S}_{1}(V) \subseteq \mathcal{S}_{2}(V) \subseteq \cdots \subseteq \mathcal{S}_{d}(V) \subseteq \cdots
$$

is the degree filtration of $V$, denoted $\mathcal{F}_{\mathrm{deg}}(V)$.

In the context of a rational subspace $V \subseteq \mathbb{F}(\lambda)^{n}$ filtered by its degree filtration, the natural bases to consider are those vector polynomial bases for $V$ that are compatible with the filtration $\mathcal{F}_{\text {deg }}(V)$, and at the same time provide $\mathcal{P}_{d}(V)$-bases for each $\mathcal{S}_{d}(V)$, as in the following definition. 
Definition 3.8 (Minimal Basis of $\mathcal{F}_{\operatorname{deg}}(V)$ ). An ordered vector polynomial basis $\mathcal{B}$ for a rational subspace $V \subseteq \mathbb{F}(\lambda)^{n}$ is said to be a minimal basis for the degree filtration $\mathcal{F}_{\mathrm{deg}}(V)$ provided

(a) $\mathcal{B}$ is compatible with the filtration $\mathcal{F}_{\operatorname{deg}}(V)$, and

(b) for each $d \geq 0$, the initial segment of $\mathcal{B}$ that forms a basis for $\mathcal{S}_{d}(V)$ is a $\mathcal{P}_{d}(V)$-basis.

Now observe that the Kronecker/Gantmacher construction (Algorithm 3.1) for generating a K-minimal basis can be simply described as follows: first find a $\mathcal{P}_{0}(V)$-basis for $\mathcal{S}_{0}(V)$, then extend to a $\mathcal{P}_{1}(V)$-basis for $\mathcal{S}_{1}(V)$, then extend to a $\mathcal{P}_{2}(V)$-basis for $\mathcal{S}_{2}(V), \ldots$, and so on inductively through the degree filtration of $V$, until a vector polynomial basis for all of $V$ is attained. But this is exactly how to generate a minimal basis for the filtration $\mathcal{F}_{\text {deg }}(V)$; indeed any minimal basis for $\mathcal{F}_{\text {deg }}(V)$ can be viewed as being generated in this way. Thus we have the following theorem.

Theorem 3.9 (Equivalence of Minimal Basis Concepts). An ordered vector polynomial basis $\mathcal{B}$ for a rational subspace $V \subseteq \mathbb{F}(\lambda)^{n}$ is a K-minimal basis for $V$ if and only if $\mathcal{B}$ is a minimal basis for the degree filtration $\mathcal{F}_{\text {deg }}(V)$.

3.3. Minimal indices from the degree filtration. In order to conveniently work with arbitrary lists of vector polynomials, we introduce one final bit of terminology.

Definition 3.10 (Degree Sequence). Suppose $\mathcal{L}=\left\{\mathrm{v}_{1}(\lambda), \mathrm{v}_{2}(\lambda), \ldots, \mathrm{v}_{k}(\lambda)\right\}$ with $d_{i}=\operatorname{deg} \mathrm{v}_{i}(\lambda)$ for $i=1, \ldots, k$ is any finite set of vector polynomials from a rational subspace $V \subseteq \mathbb{F}(\lambda)^{n}$, and let $\mathcal{L}$ be ordered so that $d_{i} \leq d_{i+1}$ for $i=1, \ldots, k-1$. Then the list of numbers $d_{1} \leq d_{2} \leq \cdots \leq d_{k}$ is the degree sequence of $\mathcal{L}$.

An important feature of the characterization in Theorem 3.9 is that it transparently reveals why every $\mathrm{K}$-minimal basis for $V$ has exactly the same list of degrees, and hence why the notion of K-minimal indices of $V$ is well-defined and meaningful. Since K-minimality and minimality with respect to the degree filtration are now seen to be equivalent notions, from now on we will just use the phrase "minimal indices," as in the following definition.

Definition 3.11 (Minimal Indices). The minimal indices of a rational subspace $V \subseteq \mathbb{F}(\lambda)^{n}$ are the numbers $\varepsilon_{1} \leq \varepsilon_{2} \leq \cdots \leq \varepsilon_{p}$ in the degree sequence of any K-minimal basis for $V$, or equivalently, the list of numbers in the degree sequence of any minimal basis for the degree filtration $\mathcal{F}_{\text {deg }}(V)$.

From this definition, it is clear that the number of minimal indices having the value $\varepsilon=0$ is just $\operatorname{dim}_{\mathbb{F}(\lambda)} \mathcal{S}_{0}(V)$, the number of minimal indices with $\varepsilon \leq 1$ is $\operatorname{dim}_{\mathbb{F}(\lambda)} \mathcal{S}_{1}(V)$, and in general for any $d \in \mathbb{N}$ the number of minimal indices with $\varepsilon \leq d$ is $\operatorname{dim}_{\mathbb{F}(\lambda)} \mathcal{S}_{d}(V)$. Thus we have a simple and intrinsic way to characterize minimal indices, which gives us the following useful tool for determining and working with them.

Theorem 3.12 (Minimal Indices from the Degree Filtration). Let $V$ be an arbitrary rational subspace of $\mathbb{F}(\lambda)^{n}$. Then the minimal indices of $V$ are uniquely determined by the dimension sequence of the degree filtration $\mathcal{F}_{\mathrm{deg}}(V)$, and vice versa. In particular, the number of zero minimal indices $\varepsilon=0$ is $\operatorname{dim}_{\mathbb{F}(\lambda)} \mathcal{S}_{0}(V)$, while for $j \geq 1$ the number of minimal indices having the value $\varepsilon=j$ is

$$
\operatorname{dim}_{\mathbb{F}(\lambda)} \mathcal{S}_{j}(V)-\operatorname{dim}_{\mathbb{F}(\lambda)} \mathcal{S}_{j-1}(V)
$$


Electronic Journal of Linear Algebra, ISSN 1081-3810

A publication of the International Linear Algebra Society

Volume 37, pp. 276-294, March 2021.

Conversely, the dimensions of the subspaces $\mathcal{S}_{d}(V)$ are uniquely determined from the minimal indices of $V$ by

$$
\operatorname{dim}_{\mathbb{F}(\lambda)} \mathcal{S}_{d}(V)=\text { "total number of minimal indices with value } \varepsilon \leq d "
$$

As an immediate corollary, we have the following criterion for deciding if two subspaces have the same minimal indices.

Corollary 3.13 (Equality of Minimal Indices). Let $\mathbb{F}$ and $\mathbb{K}$ be any two fields, and let $n$ and $q$ be any two positive integers. Then a pair of subspaces $V \subseteq \mathbb{F}(\lambda)^{n}$ and $W \subseteq \mathbb{K}(\lambda)^{q}$ have the same minimal indices if and only if

$$
\operatorname{dim}_{\mathbb{F}(\lambda)} \mathcal{F}_{\operatorname{deg}}(V)=\operatorname{dim}_{\mathbb{K}(\lambda)} \mathcal{F}_{\operatorname{deg}}(W)
$$

i.e., if and only if the degree filtrations of $V$ and $W$ have identical dimension sequences.

Another significant corollary of Theorem 3.12 is the characterization of minimal bases by their degree sequence.

Corollary 3.14 (Degree Sequence Characterizes Minimal Bases). Suppose $\mathcal{B}$ is any vector polynomial basis for a rational subspace $V \subseteq \mathbb{F}(\lambda)^{n}$. Then $\mathcal{B}$ is a minimal basis for the degree filtration $\mathcal{F}_{\mathrm{deg}}(V)$ if and only if its degree sequence is identical to the minimal indices of $V$.

Proof. The $(\Rightarrow)$ direction follows immediately from Definition 3.11 and Theorem 3.12. For the converse $(\Leftarrow)$, consider a vector polynomial basis $\mathcal{B}$, ordered as in Definition 3.10, whose degree sequence exactly matches the minimal indices of $V$. Then for $d=0$, the number of vectors in $\mathcal{B}$ with degree zero is exactly the same as $\operatorname{dim}_{\mathbb{F}(\lambda)} \mathcal{S}_{0}(V)$. Thus an initial segment of $\mathcal{B}$ forms a $\mathcal{P}_{0}(V)$-basis for $\mathcal{S}_{0}(V)$. Indeed, by $(3.11)$ we see that for each $d \geq 0$, there is an initial segment of $\mathcal{B}$ that forms a $\mathcal{P}_{d}(V)$-basis for $\mathcal{S}_{d}(V)$. Hence, $\mathcal{B}$ forms a minimal basis for $\mathcal{F}_{\text {deg }}(V)$ by Definition 3.8, i.e., $\mathcal{B}$ is a minimal basis for $V$ in the filtration sense.

REMARK 3.15. It is interesting to note that the successive-difference calculation used in (3.10) of Theorem 3.12 is also important in other filtrations. For example, consider the dimension sequence of the Jordan filtration associated with an eigenvalue $\lambda_{0}$, as described in Example 2.3(d). Then successive differences of dimensions as in (3.10) yield the Weyr characteristic of $\lambda_{0}$ [21]; taking the conjugate partition (see [21] or [23]) of the Weyr characteristic gives the Segre characteristic of $\lambda_{0}$.

The analogous successive-difference calculation on the dimension sequence of the Brunovsky filtration associated with a matrix pair $(A, B)$ (see Example 2.3(e)) produces a sequence of numbers sometimes referred to as the Brunovsky indices [2] of $(A, B)$; the conjugate partition of this Brunovsky index sequence then yields the controllability indices of $(A, B)[23]$.

In Sections 5 and 6, we will illustrate the efficacy of the tools provided by Theorem 3.12 and Corollary 3.13 by establishing two fundamental properties of minimal indices:

(i) for nullspaces of matrix polynomials, minimal indices are unchanged by passage to any field extension,

(ii) minimal indices behave nicely under direct sums; i.e., the list of minimal indices of $V \oplus W$, where $V \subseteq \mathbb{F}(\lambda)^{n}$ and $W \subseteq \mathbb{F}(\lambda)^{m}$, is just the concatenation of the list of minimal indices of $V$ with the list of minimal indices of $W$. 
But first we will see how the degree filtration notion of minimal indices connects up with Forney's approach in [7]. This connection will be made via a property of minimal indices brought to light by the filtration view, a "strong" minimality property that leads to a characterization of the vector polynomial bases in any rational subspace of $\mathbb{F}(\lambda)^{n}$.

4. A strong minimality property of minimal indices. The filtration view of minimal indices provides insight that is not so readily obtained from either the Kronecker or Forney point of view, as illustrated by the following simple example.

Example 4.1. Suppose a two-dimensional rational subspace $V \subseteq \mathbb{F}(\lambda)^{n}$ has minimal indices $\varepsilon_{1}=1$ and $\varepsilon_{2}=4$. Is it possible for there to exist a vector polynomial basis $\mathcal{B}=\left\{\mathrm{v}_{1}(\lambda), \mathrm{v}_{2}(\lambda)\right\}$ for $V$ with degree sequence $(3,3)$ ? From the Kronecker/Gantmacher point of view, this seems to be perfectly plausible; once you choose $\mathrm{v}_{1}(\lambda)$ with non-minimal degree $\left(\operatorname{deg} \mathrm{v}_{1}=3\right)$ to be the first basis vector in $\mathcal{B}$, then you are no longer following the greedy algorithm, so all bets are off as to what might be available for a second basis vector. The Forney view also does not seem to offer any objection to the existence of such a basis $\mathcal{B}$, since $\operatorname{ord}(\mathcal{B})=6$ is certainly compatible with the minimal order being $\varepsilon_{1}+\varepsilon_{2}=5$.

In fact, though, it is impossible for $V$ to have such a basis $\mathcal{B}$, and this can be seen rather easily from the filtration point of view. The minimal indices $\varepsilon_{1}=1$ and $\varepsilon_{2}=4$ immediately imply that the dimension sequence of the degree filtration of $V$ must be $(0,1,1,1,2,2,2,2, \ldots)$; in particular, we would have $\operatorname{dim} \mathcal{S}_{3}(V)=1$. But the existence of a vector polynomial basis $\mathcal{B}$ with degree sequence $(3,3)$ would mean that $\mathcal{S}_{3}(V)$ would have to have dimension 2. This contradiction shows the impossibility of such a basis $\mathcal{B}$ for $V$. This example also hints at the presence of subtle constraints on the possible vector polynomial bases in a general rational vector space $V \subseteq \mathbb{F}(\lambda)^{n}$, or at least at constraints that are not so obvious from either the Forney or Kronecker/Gantmacher definitions.

The idea used to resolve the question posed in Example 4.1 can be extended and refined to establish the following strong minimality property (4.12), which now characterizes the degree sequences of all vector polynomial bases in any rational subspace $V \subseteq \mathbb{F}(\lambda)^{n}$, and thus fully justifies the name "minimal indices". Note that the proof given here is a modified version of an argument used in [3, Lemma 2.4].

Theorem 4.2 (Strong Minimality Property of Minimal Indices). Suppose that $V \subseteq \mathbb{F}(\lambda)^{n}$ is a pdimensional rational subspace with minimal indices $\varepsilon_{1} \leq \varepsilon_{2} \leq \cdots \leq \varepsilon_{p}$. Then $\delta_{1} \leq \delta_{2} \leq \cdots \leq \delta_{p}$ is the degree sequence of some vector polynomial basis $\mathcal{B}=\left\{\mathrm{v}_{1}(\lambda), \ldots, \mathrm{v}_{p}(\lambda)\right\}$ for $V$ if and only if

$$
\varepsilon_{j} \leq \delta_{j} \quad \text { for each } j=1, \ldots, p
$$

Proof. ( $\Rightarrow$ ) If $\delta_{1} \leq \delta_{2} \leq \cdots \leq \delta_{p}$ is the degree sequence of $\mathcal{B}$, then clearly $\varepsilon_{1} \leq \delta_{1}$, since $\varepsilon_{1}$ is the minimal degree among all vector polynomials in $V$. Now suppose (4.12) fails for some $1<j \leq p$, and let $m$ be the smallest such $j$. With $d:=\varepsilon_{m}-1$, we would then have

$$
\varepsilon_{m-1} \leq \delta_{m-1} \leq \delta_{m} \leq d<\varepsilon_{m}
$$

Now by Theorem 3.12, $\varepsilon_{m-1} \leq d<\varepsilon_{m}$ implies that $\operatorname{dim} \mathcal{S}_{d}(V)=m-1$. But $\delta_{m} \leq d$ means that $\left\{\mathrm{v}_{1}(\lambda), \ldots, \mathrm{v}_{m}(\lambda)\right\}$ would be a linearly independent set of vector polynomials in $\mathcal{S}_{d}(V)$, forcing $\operatorname{dim} \mathcal{S}_{d}(V) \geq$ $m$. This contradiction shows that (4.12) cannot fail at any $j$. 
$(\Leftarrow)$ To see that any degree sequence $\delta_{1} \leq \delta_{2} \leq \cdots \leq \delta_{p}$ satisfying (4.12) can be realized by some vector polynomial basis for $V$, let $\left\{\mathrm{w}_{1}(\lambda), \mathrm{w}_{2}(\lambda), \ldots, \mathrm{w}_{p}(\lambda)\right\}$ be any minimal basis for $V$, with degree sequence $\varepsilon_{1} \leq \varepsilon_{2} \leq \cdots \leq \varepsilon_{p}$. Then

$$
\mathcal{B}:=\left\{\lambda^{\delta_{1}-\varepsilon_{1}} \mathbf{w}_{1}(\lambda), \lambda^{\delta_{2}-\varepsilon_{2}} \mathbf{w}_{2}(\lambda), \ldots, \lambda^{\delta_{p}-\varepsilon_{p}} \mathbf{w}_{p}(\lambda)\right\}
$$

is clearly a vector polynomial basis for $V$, with the desired degree sequence $\delta_{1} \leq \delta_{2} \leq \cdots \leq \delta_{p}$.

As an easy consequence of this strong minimality property, we can now immediately (and simultaneously) establish two of the fundamental properties of the F-minimal indices introduced by Forney in [7]: that they are well-defined, and that they are identical to the K-minimal indices. Note that this argument is completely independent of the results in [7], and substantially simplifies and unifies our understanding of the relationships between these various notions of minimal indices.

Corollary 4.3 (F-minimal and K-minimal Indices are Identical). A vector polynomial basis $\mathcal{B}$ for a rational subspace $V \subseteq \mathbb{F}(\lambda)^{n}$ is F-minimal (i.e., has minimal order) if and only if its degree sequence is identical to the minimal indices of the degree filtration $\mathcal{F}_{\mathrm{deg}}(V)$.

Proof. By (4.12) in Theorem 4.2 it is clear that for every vector polynomial basis $\mathcal{B}$ for $V$, the order of $\mathcal{B}$ is bounded below by the sum $\mu=\sum_{j} \varepsilon_{j}$ of all the minimal indices of the degree filtration $\mathcal{F}_{\text {deg }}(V)$. But since this lower bound $\mu$ is actually attained by any K-minimal basis, this must be the order of any F-minimal basis for $V$. It is also clear from (4.12) that the only way for a vector polynomial basis $\mathcal{B}$ to attain the minimum order $\mu$ is for its degree sequence to be identical to the minimal indices of the degree filtration $\mathcal{F}_{\text {deg }}(V)$.

REMARK 4.4. The strong minimality property of minimal indices proved in Theorem 4.2 does not seem to be widely known. However, it does appear in the coding theory literature, as least as early as [18].

5. Minimal indices and field extensions. Historically, the original reason to introduce the notions of minimal bases and indices was to help clarify the properties of the Kronecker canonical form (KCF), a classical result for matrix pencils over algebraically closed fields (see [9]). In a recent investigation of a new equivalence relation on matrix polynomials [4], matrix pencils $L(\lambda)$ over an arbitrary field $\mathbb{F}$ were under consideration, and we wanted to make use of the KCF of $L(\lambda)$, viewed as a pencil over the algebraic closure $\overline{\mathbb{F}}$. In this context, a key question is whether the minimal indices of $L$ can be affected by the change of field from $\mathbb{F}$ to $\overline{\mathbb{F}}$. The goal of this section is to resolve this issue, by proving the following invariance result for the minimal indices of a matrix polynomial over an arbitrary field.

ThEOREM 5.1 (Invariance of Minimal Indices under Field Extension). Suppose P( $\lambda$ ) is an $m \times n$ matrix polynomial over a field $\mathbb{F}$, and $\widetilde{\mathbb{F}} \supseteq \mathbb{F}$ is an extension field. Let

$$
V:=\left\{\mathrm{v}(\lambda) \in \mathbb{F}(\lambda)^{n}: P(\lambda) \mathrm{v}(\lambda) \equiv 0\right\} \subseteq \mathbb{F}(\lambda)^{n}
$$

be the right nullspace of $P(\lambda)$ viewed as a matrix polynomial over $\mathbb{F}$, and let

$$
W:=\left\{\mathrm{w}(\lambda) \in \widetilde{\mathbb{F}}(\lambda)^{n}: P(\lambda) \mathrm{w}(\lambda) \equiv 0\right\} \subseteq \widetilde{\mathbb{F}}(\lambda)^{n},
$$

be the right nullspace of $P(\lambda)$ viewed as a matrix polynomial over $\widetilde{\mathbb{F}}$. Then the minimal indices of $V$ and $W$ are identical.

The strategy of the proof is to show that the dimension sequences $\operatorname{dim} \mathcal{F}_{\text {deg }}(V)$ over the field $\mathbb{F}(\lambda)$, and $\operatorname{dim} \mathcal{F}_{\text {deg }}(W)$ over the field $\widetilde{\mathbb{F}}(\lambda)$, are identical; Corollary 3.13 then implies the desired conclusion. Before we get to the proof of the theorem, we need some preliminary results. 
Lemma 5.2. Suppose $\mathbb{K}$ is a field, $\widetilde{\mathbb{K}} \supseteq \mathbb{K}$ is a field extension, and $\mathrm{v}_{1}, \mathrm{v}_{2}, \ldots, \mathrm{v}_{\ell} \in \mathbb{K}^{n} \subseteq \widetilde{\mathbb{K}}^{n}$ are column vectors in $\mathbb{K}^{n}$ (hence also in $\widetilde{\mathbb{K}}^{n}$ ). Then

$\mathrm{v}_{1}, \mathrm{v}_{2}, \ldots, \mathrm{v}_{\ell}$ are linearly independent in $\mathbb{K}^{n} \Longleftrightarrow \mathrm{v}_{1}, \mathrm{v}_{2}, \ldots, \mathrm{v}_{\ell}$ are linearly independent in $\widetilde{\mathbb{K}}^{n}$.

Proof. Line up the $\ell$ column vectors $\mathrm{v}_{1}, \mathrm{v}_{2}, \ldots, \mathrm{v}_{\ell}$ side-by-side to form an $n \times \ell$ matrix

$$
A=\left[\mathrm{v}_{1} \mathrm{v}_{2} \ldots \mathrm{v}_{\ell}\right] \in \mathbb{K}^{n \times \ell} \subset \widetilde{\mathbb{K}}^{n \times \ell}
$$

Then $\mathrm{v}_{1}, \mathrm{v}_{2}, \ldots, \mathrm{v}_{\ell}$ are linearly independent in $\mathbb{K}^{n} \Leftrightarrow A$ has an $\ell \times \ell$ submatrix $\widehat{A}$ such that $\operatorname{det} \widehat{A} \neq 0 \Leftrightarrow$ $\mathrm{v}_{1}, \mathrm{v}_{2}, \ldots, \mathrm{v}_{\ell}$ are linearly independent in $\widetilde{\mathbb{K}}^{n}$.

Lemma 5.3. Let $P(\lambda), V$, and $W$ be as in Theorem 5.1. Then for each $d \in \mathbb{N}$, we have

$$
\operatorname{dim}_{\mathbb{F}} \mathcal{P}_{d}(V)=\operatorname{dim}_{\widetilde{\mathbb{F}}} \mathcal{P}_{d}(W)
$$

Proof. Let $P(\lambda)=A_{0}+\lambda A_{1}+\lambda^{2} A_{2}+\cdots+\lambda^{k} A_{k}$ be an $m \times n$ matrix polynomial over a field $\mathbb{F}$. Extending a technique used by Gantmacher [9] to analyze singular pencils, consider the following (possibly rectangular) block-Toeplitz matrices built from the coefficient matrices of $P(\lambda)$ :

$$
M_{0}:=\left[\begin{array}{c}
A_{0} \\
A_{1} \\
\vdots \\
A_{k}
\end{array}\right], \quad M_{1}:=\left[\begin{array}{cc}
A_{0} & 0 \\
A_{1} & A_{0} \\
\vdots & A_{1} \\
A_{k} & \vdots \\
0 & A_{k}
\end{array}\right], \ldots, \quad M_{d}:=\left[\begin{array}{cccc}
A_{0} & & & \\
A_{1} & A_{0} & & \\
\vdots & A_{1} & \ddots & \\
A_{k} & \vdots & \ddots & A_{0} \\
& A_{k} & & A_{1} \\
& & \ddots & \vdots \\
& & & A_{k}
\end{array}\right], \ldots
$$

where $M_{d}$ has $d+1$ block columns. Now suppose $\mathrm{v}(\lambda)=\mathrm{v}_{0}+\lambda \mathrm{v}_{1}+\lambda^{2} \mathrm{v}_{2}+\cdots+\lambda^{d} \mathrm{v}_{d}$ is a vector polynomial of degree at most $d$ with $\mathrm{v}_{i} \in \mathbb{F}^{n}$ for $i=0, \ldots, d$, and $\widetilde{\mathrm{v}}:=\left[\mathrm{v}_{0}^{T} \mathrm{v}_{1}^{T} \ldots \mathrm{v}_{d}^{T}\right]^{T}$ is the vector in $\mathbb{F}^{(d+1) n}$ formed by vertically stacking up all the coefficient vectors $\vee_{i}$. Then it is not hard to see that $\mathrm{v}(\lambda) \in \mathcal{P}_{d}(V)$ if and only if

$$
A_{0} \mathrm{v}_{0}=0, \quad A_{1} \mathrm{v}_{0}+A_{0} \mathrm{v}_{1}=0, A_{2} \mathrm{v}_{0}+A_{1} \mathrm{v}_{1}+A_{0} \mathrm{v}_{2}=0, \ldots, A_{k} \mathrm{v}_{d}=0
$$

if and only if $M_{d} \widetilde{v}=0$. From this it follows that the map

$$
\begin{aligned}
\mathcal{P}_{d}(V) & \longrightarrow \operatorname{ker} M_{d} \\
\mathrm{v}(\lambda) & \longmapsto \widetilde{\mathrm{v}},
\end{aligned}
$$

is a linear isomorphism of $\mathbb{F}$-vector spaces, and so $\operatorname{dim}_{\mathbb{F}} \mathcal{P}_{d}(V)=\operatorname{dim}_{\mathbb{F}} \operatorname{ker} M_{d}$.

The same argument, viewing $M_{d}$ and the coefficients of $P(\lambda)$ as matrices with entries in any extension field $\widetilde{\mathbb{F}}$, shows that $\operatorname{dim}_{\widetilde{\mathbb{F}}} \mathcal{P}_{d}(W)=\operatorname{dim}_{\widetilde{\mathbb{F}}} \operatorname{ker} M_{d}$. Now the rank/nullity theorem implies that $\operatorname{dim} \operatorname{ker} M_{d}=$ $(d+1) n-\operatorname{rank} M_{d}$. But the rank of a matrix is the size of the largest square submatrix with nonzero determinant, so rank is insensitive to field extensions. Thus we have

$$
\operatorname{dim}_{\mathbb{F}} \mathcal{P}_{d}(V)=\operatorname{dim}_{\mathbb{F}} \operatorname{ker} M_{d}=(d+1) n-\operatorname{rank} M_{d}=\operatorname{dim}_{\widetilde{\mathbb{F}}} \operatorname{ker} M_{d}=\operatorname{dim}_{\widetilde{\mathbb{F}}} \mathcal{P}_{d}(W),
$$

and the proof is complete. 
With these two lemmas in hand, we now return to the proof of Theorem 5.1.

Proof of Theorem 5.1. The strategy of the proof is to show that for each $d \in \mathbb{N}$, the $\mathbb{F}(\lambda)$-dimension of $\mathcal{S}_{d}(V)$ is the same as the $\widetilde{\mathbb{F}}(\lambda)$-dimension of $\mathcal{S}_{d}(W)$, and hence that

$$
\operatorname{dim}_{\mathbb{F}(\lambda)} \mathcal{F}_{\operatorname{deg}}(V)=\operatorname{dim}_{\widetilde{\mathbb{F}}(\lambda)} \mathcal{F}_{\operatorname{deg}}(W)
$$

i.e., the degree filtrations $\mathcal{F}_{\operatorname{deg}}(V)$ and $\mathcal{F}_{\text {deg }}(W)$ have identical dimension sequences. It then follows from Corollary 3.13 that the minimal indices of $V$ and $W$ are identical, as desired.

To get a handle on the spaces $\mathcal{S}_{d}(V)$ and $\mathcal{S}_{d}(W)$, we begin by considering the vector polynomial spaces $\mathcal{P}_{d}(V)$ and $\mathcal{P}_{d}(W)$. Let $\mathcal{B}$ be any $\mathbb{F}$-basis for the $\mathbb{F}$-vector space $\mathcal{P}_{d}(V)$. Then clearly $\mathcal{B} \subseteq \mathcal{P}_{d}(W)$, and by Lemma 5.2 (with $\mathbb{K}=\mathbb{F}$ and $\widetilde{\mathbb{K}}=\widetilde{\mathbb{F}}$ ) it follows that $\mathcal{B}$ is an $\widetilde{\mathbb{F}}$-linearly independent subset of $\mathcal{P}_{d}(W$ ). The equality of the dimensions $\operatorname{dim}_{\mathbb{F}} \mathcal{P}_{d}(V)$ and $\operatorname{dim}_{\widetilde{\mathbb{F}}} \mathcal{P}_{d}(W)$ from Lemma 5.3 now implies that $\mathcal{B}$ is also an $\widetilde{\mathbb{F}}$-basis for the $\widetilde{\mathbb{F}}$-vector space $\mathcal{P}_{d}(W)$.

Since $\mathcal{P}_{d}(V)$ is $\mathbb{F}$-generated by $\mathcal{B}$, and $\mathcal{S}_{d}(V)$ is $\mathbb{F}(\lambda)$-generated by $\mathcal{P}_{d}(V)$, the fact that $\mathbb{F} \subseteq \mathbb{F}(\lambda)$ implies that $\mathcal{B}$ is an $\mathbb{F}(\lambda)$-spanning set for $\mathcal{S}_{d}(V)$. The same kind of argument shows that $\mathcal{B}$ is also an $\widetilde{\mathbb{F}}(\lambda)$-spanning set for $\mathcal{S}_{d}(W)$.

Inside of the spanning set $\mathcal{B}$ we can now find a subset $\widehat{\mathcal{B}} \subseteq \mathcal{B}$ that forms an $\mathbb{F}(\lambda)$-basis for $\mathcal{S}_{d}(V)$, and hence is a maximal $\mathbb{F}(\lambda)$-linearly independent subset of $\mathcal{S}_{d}(V)$. Using Lemma 5.2 again, this time with $\mathbb{K}=\mathbb{F}(\lambda)$ and $\widetilde{\mathbb{K}}=\widetilde{\mathbb{F}}(\lambda)$, we see that $\widehat{\mathcal{B}}$ is not only an $\widetilde{\mathbb{F}}(\lambda)$-linearly independent subset of $\mathcal{S}_{d}(W)$, it is actually a maximal $\widetilde{\mathbb{F}}(\lambda)$-linearly independent subset of $\mathcal{S}_{d}(W)$. [ If not, then there would be a strictly larger $\widetilde{\mathbb{F}}(\lambda)$-linearly independent subset $\mathcal{B}^{\prime}$ of $\mathcal{B}$, i.e., $\widehat{\mathcal{B}} \subset \mathcal{B}^{\prime} \subseteq \mathcal{B} \subseteq \mathcal{P}_{d}(V)$, which by Lemma 5.2 would contradict the maximality of $\widehat{\mathcal{B}}$ as an $\mathbb{F}(\lambda)$-linearly independent subset of $\mathcal{S}_{d}(V)$. ] Thus, we see that $\widehat{\mathcal{B}}$ is simultaneously an $\mathbb{F}(\lambda)$-basis for $\mathcal{S}_{d}(V)$ as well as an $\widetilde{\mathbb{F}}(\lambda)$-basis for $\mathcal{S}_{d}(W)$, showing that $\operatorname{dim}_{\mathbb{F}(\lambda)} \mathcal{S}_{d}(V)=\operatorname{dim}_{\widetilde{\mathbb{F}}(\lambda)} \mathcal{S}_{d}(W)$, and the proof is complete.

REMARK 5.4. Note that Theorem 5.1, despite the simple nature of its statement, has not to our knowledge appeared before in the literature. Indeed, the search for a clear proof of this result was the primary motivation for developing the filtration formulation of minimal indices in the first place.

REMARK 5.5. The invariance of minimal indices under field extension also holds for the left nullspace of any matrix polynomial $P(\lambda)$. Simply apply Theorem 5.1 to the right nullspace of $P^{T}(\lambda)$, which is the same as the left nullspace of $P(\lambda)$.

6. Minimal indices and direct sums. In the course of developing the Kronecker canonical form in [9], Gantmacher remarks without proof that:

The complete system of indices for the columns (rows) of a quasi-diagonal matrix is obtained as the union of the corresponding systems of minimal indices of the individual diagonal blocks. [9, Vol. II, p. 39]

In my view, this assertion is not obvious and requires some proof, especially since it is an essential component of the overall argument for the KCF. In this section, we close this "gap" by showing that minimal indices behave nicely under direct sums more generally; in particular, we show that the list of minimal indices of $V \oplus W$ is just the concatenation of the individual lists of minimal indices of $V$ and $W$. The filtration view of minimal indices allows for a completely straightforward proof of this result, although some preliminary 
discussion of how filtrations interact with direct sums is needed. Thus we begin with the simple notion of the direct sum of filtrations, applicable to any pair of filtered vector spaces.

Definition 6.1 (Direct Sum of Filtrations). Suppose $V$ and $W$ are filtered vector spaces over the same field $\mathbb{K}$, with given filtrations

$$
\mathcal{F}(V): V_{0} \subseteq V_{1} \subseteq V_{2} \subseteq \cdots \quad \text { and } \quad \mathcal{F}(W): W_{0} \subseteq W_{1} \subseteq W_{2} \subseteq \cdots,
$$

respectively. Then the direct sum filtration $\mathcal{F}(V) \oplus \mathcal{F}(W)$ of the $\mathbb{K}$-vector space $V \oplus W$ is defined to be

$$
V_{0} \oplus W_{0} \subseteq V_{1} \oplus W_{1} \subseteq V_{2} \oplus W_{2} \subseteq \cdots
$$

In Section 3 we introduced some special vector spaces $V$ having a canonical filtration, namely subspaces $V \subseteq \mathbb{F}(\lambda)^{n}$ equipped with the degree filtration $\mathcal{F}_{\text {deg }}(V)$. An obvious question is whether the degree filtration of the direct sum $V \oplus W$ has any nice relationship to the degree filtrations of the individual spaces $V$ and $W$. The next result gives the simple answer.

Lemma 6.2 (Degree Filtration of Direct Sum). Let $V \subseteq \mathbb{F}(\lambda)^{n}$ and $W \subseteq \mathbb{F}(\lambda)^{k}$ be arbitrary rational subspaces, so that $V \oplus W$ is a rational subspace of $\mathbb{F}(\lambda)^{n+k}$. Then

$$
\mathcal{F}_{\text {deg }}(V \oplus W)=\mathcal{F}_{\operatorname{deg}}(V) \oplus \mathcal{F}_{\operatorname{deg}}(W)
$$

Proof. To determine the degree filtration of $V \oplus W$, we must first understand the vector polynomial subsets $\mathcal{P}_{d}(V \oplus W)$. Any element of $V \oplus W$ has the form $z=\left[\begin{array}{c}v \\ w\end{array}\right]$, where $v \in V$ and $w \in W$. Thus any vector polynomial $\mathrm{z} \in \mathcal{P}_{d}(V \oplus W)$ must be built from some $\mathrm{v} \in \mathcal{P}_{d}(V)$ together with some $\mathrm{w} \in \mathcal{P}_{d}(W)$. Consequently we see that $\mathcal{P}_{d}(V \oplus W)=\mathcal{P}_{d}(V) \oplus \mathcal{P}_{d}(W)$.

Next we consider the $\mathbb{F}(\lambda)$-span of $\mathcal{P}_{d}(V \oplus W)$, or equivalently of $\mathcal{P}_{d}(V) \oplus \mathcal{P}_{d}(W)$, in order to obtain the $\mathbb{F}(\lambda)$-subspace $\mathcal{S}_{d}(V \oplus W)$ that is part of the degree filtration $\mathcal{F}_{\operatorname{deg}}(V \oplus W)$. It is easy to see that any linear combination of elements $z_{i}$ from the direct sum $\mathcal{P}_{d}(V) \oplus \mathcal{P}_{d}(W)$ can be expressed as the direct sum of a linear combination from $\mathcal{P}_{d}(V)$ together with a linear combination from $\mathcal{P}_{d}(W)$ :

$$
\sum_{i} c_{i} \mathbf{z}_{i}=\sum_{i} c_{i}\left[\begin{array}{c}
\mathbf{v}_{i} \\
\mathbf{w}_{i}
\end{array}\right]=\left[\begin{array}{c}
\sum c_{i} \mathbf{v}_{i} \\
\sum c_{i} \mathbf{w}_{i}
\end{array}\right]=\sum_{i} c_{i} \mathbf{v}_{i} \oplus \sum_{i} c_{i} \mathrm{w}_{i} .
$$

Conversely, any direct sum of a linear combination from $\mathcal{P}_{d}(V)$ and a linear combination from $\mathcal{P}_{d}(W)$ can be written as a linear combination of elements from $\mathcal{P}_{d}(V) \oplus \mathcal{P}_{d}(W)$ :

$$
\begin{aligned}
\sum_{i} b_{i} \mathrm{v}_{i} \oplus \sum_{j} c_{j} \mathrm{w}_{j} & =\left[\begin{array}{c}
\sum b_{i} \mathrm{v}_{i} \\
\sum c_{j} \mathrm{w}_{j}
\end{array}\right]=\left[\begin{array}{c}
\sum b_{i} \mathrm{v}_{i} \\
0
\end{array}\right]+\left[\begin{array}{c}
0 \\
\sum c_{j} \mathrm{w}_{j}
\end{array}\right] \\
& =\sum_{i} b_{i}\left[\begin{array}{c}
\mathrm{v}_{i} \\
0
\end{array}\right]+\sum_{j} c_{j}\left[\begin{array}{c}
0 \\
\mathrm{w}_{j}
\end{array}\right] .
\end{aligned}
$$

Together these observations imply that

$$
\underset{\mathbb{F}(\lambda)}{\operatorname{span}}\left[\mathcal{P}_{d}(V) \oplus \mathcal{P}_{d}(W)\right]=\underset{\mathbb{F}(\lambda)}{\operatorname{span}}\left[\mathcal{P}_{d}(V)\right] \oplus \underset{\mathbb{F}(\lambda)}{\operatorname{span}}\left[\mathcal{P}_{d}(W)\right]=: \mathcal{S}_{d}(V) \oplus \mathcal{S}_{d}(W) .
$$


Thus we have

$$
\mathcal{S}_{d}(V \oplus W):=\operatorname{span}_{\mathbb{F}(\lambda)}\left[\mathcal{P}_{d}(V \oplus W)\right]=\underset{\mathbb{F}(\lambda)}{\operatorname{span}}\left[\mathcal{P}_{d}(V) \oplus \mathcal{P}_{d}(W)\right]=\mathcal{S}_{d}(V) \oplus \mathcal{S}_{d}(W)
$$

Assembling these together for all $d \in \mathbb{N}$ shows that (6.14) holds.

Now that we know how degree filtrations behave with respect to direct sums, it is just one easy further step to see how the minimal indices of a direct sum are related to the minimal indices of the summands.

TheOREm 6.3 (Minimal Indices of Direct Sum). Let $V \subseteq \mathbb{F}(\lambda)^{n}$ and $W \subseteq \mathbb{F}(\lambda)^{k}$ be arbitrary rational subspaces, so that $V \oplus W$ is a subspace of $\mathbb{F}(\lambda)^{n+k}$. Then the list of minimal indices of $V \oplus W$ is just the concatenation of the list of minimal indices of $V$ together with the list of those of $W$.

Proof. From (6.14) it follows that the dimension sequence of $\mathcal{F}_{\text {deg }}(V \oplus W)$ is just the (entry-wise) sum of the dimension sequences of $\mathcal{F}_{\mathrm{deg}}(V)$ and $\mathcal{F}_{\mathrm{deg}}(W)$, i.e., that

$$
\operatorname{dim} \mathcal{F}_{\text {deg }}(V \oplus W)=\operatorname{dim} \mathcal{F}_{\text {deg }}(V)+\operatorname{dim} \mathcal{F}_{\text {deg }}(W)
$$

The desired result now follows from (6.15) and the minimal index formula (3.10) in Theorem 3.12:

$$
\begin{aligned}
\#(\min . \text { indices } \varepsilon=d \text { for } V \oplus W) & =\operatorname{dim} \mathcal{S}_{d}(V \oplus W)-\operatorname{dim} \mathcal{S}_{d-1}(V \oplus W) \\
& =\left\{\begin{array}{c}
{\left[\operatorname{dim} \mathcal{S}_{d}(V)+\operatorname{dim} \mathcal{S}_{d}(W)\right]} \\
-\left[\operatorname{dim} \mathcal{S}_{d-1}(V)+\operatorname{dim} \mathcal{S}_{d-1}(W)\right]
\end{array}\right\} \\
& =\left\{\begin{array}{c}
{\left[\operatorname{dim} \mathcal{S}_{d}(V)-\operatorname{dim} \mathcal{S}_{d-1}(V)\right]} \\
+\left[\operatorname{dim} \mathcal{S}_{d}(W)-\operatorname{dim} \mathcal{S}_{d-1}(W)\right]
\end{array}\right\} \\
& =\left\{\begin{array}{c}
\#(\min . \text { indices } \varepsilon=d \text { for } V) \\
+\#(\min . \text { indices } \varepsilon=d \text { for } W)
\end{array}\right\},
\end{aligned}
$$

and the theorem is proved.

7. Further properties of minimal bases. In this final section we consider two additional significant properties of minimal bases, the polynomial linear combination property and the predictable degree property. Although these properties were previously established using Forney's definition [7], we include a discussion of them here to further illustrate how the filtration point of view leads to natural and straightforward proofs, as well as provides new insight into the properties themselves. Note that we only outline these filtration-based proofs, leaving some of the details to the reader.

The first of these properties concerns the generation of vector polynomials from a vector polynomial basis of a rational subspace $V \subseteq \mathbb{F}(\lambda)^{n}$.

Definition 7.1 (Polynomial Linear Combination Property). Suppose $V \subseteq \mathbb{F}(\lambda)^{n}$ is a rational subspace, and $\mathcal{B}=\left\{\mathbf{v}_{j}(\lambda)\right\}_{j=1, k}$ is a vector polynomial basis for $V$, so that every $\mathbf{w}(\lambda) \in V$ is uniquely expressed as an $\mathbb{F}(\lambda)$-linear combination

$$
\mathrm{w}(\lambda)=\sum_{j=1}^{k} r_{j}(\lambda) \mathrm{v}_{j}(\lambda), \quad \text { with } \quad r_{j}(\lambda) \in \mathbb{F}(\lambda)
$$


Then we say that the basis $\mathcal{B}$ has the polynomial linear combination (PLC) property if for every vector polynomial $\mathrm{w}(\lambda) \in V$, the rational coefficients $r_{j}(\lambda)$ in (7.16) are all polynomials. In other words, every vector polynomial in $V$ can be generated as a polynomial linear combination of the vectors in $\mathcal{B}$.

Theorem 7.2 (Minimal Bases have the PLC Property). Any minimal basis $\mathcal{B}$ (in the filtration sense) for a rational subspace $V \subseteq \mathbb{F}(\lambda)^{n}$ has the PLC property.

Proof. One nice feature of the filtration view of minimal bases is that it encourages and facilitates the use of induction arguments moving up through the degree filtration $\mathcal{F}_{\text {deg }}(V)$ of $V$. Indeed, this Theorem 7.2 may be proved using exactly that strategy.

Begin by establishing two simple background facts concerning proper rational functions $r_{i}(\lambda)$ (i.e., rational functions $\frac{u(\lambda)}{\ell(\lambda)}$ where $\operatorname{deg} u<\operatorname{deg} \ell$ ), and proper rational linear combinations of vector polynomials $\mathbf{v}_{i}(\lambda)$ with $\operatorname{deg} \mathbf{v}_{i} \leq k:$

(F1) $q(\lambda):=\sum_{i}^{m} r_{i}(\lambda)$ is a polynomial $\Rightarrow q=0$.

(F2) $\mathbf{w}(\lambda):=\sum_{i}^{m} r_{i}(\lambda) \mathbf{v}_{i}(\lambda)$ is a vector polynomial $\Rightarrow \operatorname{deg} \mathbf{w}<k$.

Then by induction on the degree parameter $d$ of the filtration $\mathcal{F}_{\text {deg }}(V)$, show that any vector polynomial $\mathbf{v}(\lambda) \in \mathcal{S}_{d}(V)$ can be expressed as a polynomial linear combination of the vectors in any $\mathcal{P}_{d}(V)$-basis for $\mathcal{S}_{d}(V)$, in particular for the $\mathcal{P}_{d}(V)$-basis $\mathcal{B}_{d} \subseteq \mathcal{B}$ forming an initial segment of the ordered basis $\mathcal{B}$.

The base case of the induction $(d=0)$ follows easily from background fact (F1). Write an arbitrary vector polynomial $\mathbf{v}(\lambda) \in \mathcal{S}_{0}(V)$ as a rational linear combination $\sum_{\mathbf{v}_{i} \in \mathcal{B}_{0}} s_{i}(\lambda) \mathbf{v}_{i}$ of (constant) vectors in $\mathcal{B}_{0}$, then expand each rational coefficient into a sum $s_{i}(\lambda)=p_{i}(\lambda)+r_{i}(\lambda)$, where $p_{i}$ is polynomial and $r_{i}$ is a proper rational function, so that

$$
\mathbf{v}(\lambda)=\sum_{\mathbf{v}_{i} \in \mathcal{B}_{0}} s_{i}(\lambda) \mathbf{v}_{i}=\sum_{\mathbf{v}_{i} \in \mathcal{B}_{0}} p_{i}(\lambda) \mathbf{v}_{i}+\sum_{\mathbf{v}_{i} \in \mathcal{B}_{0}} r_{i}(\lambda) \mathbf{v}_{i}
$$

The rightmost sum in (7.17) can now be seen to be the zero vector, using (F1) on each component. Thus the PLC property holds for the minimal basis $\mathcal{B}_{0}$ of the rational subspace $\mathcal{S}_{0}(V)$.

For a general $d$, we can proceed in a similar manner and consider an arbitrary vector polynomial $\mathbf{v}(\lambda) \in \mathcal{S}_{d}(V)$, written as a rational linear combination of vector polynomials in $\mathcal{B}_{d}$. Again expanding each of these rational coefficients as a sum of a polynomial and a proper rational function, we have

$$
\mathbf{v}(\lambda)=\sum_{\mathbf{v}_{j} \in \mathcal{B}_{d}} s_{j}(\lambda) \mathbf{v}_{j}(\lambda)=\sum_{\mathbf{v}_{j} \in \mathcal{B}_{d}} p_{j}(\lambda) \mathbf{v}_{j}(\lambda)+\underbrace{\sum_{\mathbf{v}_{j} \in \mathcal{B}_{d}} r_{j}(\lambda) \mathbf{v}_{j}(\lambda)}_{\mathbf{w}(\lambda)} .
$$

Using fact (F2), we can now see that the rightmost sum in (7.18) represents a vector polynomial $\mathbf{w}(\lambda)$ in $V$ of degree less than $d$, and hence in $\mathcal{P}_{d-1}(V) \subseteq \mathcal{S}_{d-1}(V)$. Then by the induction hypothesis this $\mathbf{w}(\lambda)$ can be expressed as a polynomial linear combination of the vectors in $\mathcal{B}_{d-1}$, and so the PLC property holds for the minimal basis $\mathcal{B}_{d}$ of the rational subspace $\mathcal{S}_{d}(V)$.

The filtration of $V$ by the subspaces $\mathcal{S}_{d}(V)$ now implies that the PLC property holds for all of $V$, completing the proof. 
The second significant property considers the question of determining the degree of polynomial linear combinations of vector polynomials, i.e., for sums of the form

$$
\mathbf{v}(\lambda):=\sum_{j=1}^{k} p_{j}(\lambda) \mathbf{v}_{j}(\lambda)
$$

where each $p_{j}(\lambda)$ is a scalar polynomial, and each $\mathbf{v}_{j}(\lambda)$ is a vector polynomial. Clearly each term $p_{j}(\lambda) \mathbf{v}_{j}(\lambda)$ has degree equal to $\left(\operatorname{deg} p_{j}+\operatorname{deg} \mathbf{v}_{j}\right)$, and the degree of the whole linear combination vector $\mathbf{v}(\lambda)$ has the upper bound

$$
\operatorname{deg} \mathbf{v}(\lambda) \leq \max _{j}\left(\left(\operatorname{deg} p_{j} \mathbf{v}_{j}\right)\right)=\max _{j}\left(\operatorname{deg} p_{j}+\operatorname{deg} \mathbf{v}_{j}\right)
$$

Indeed, we might reasonably expect equality to hold generically in this bound, since for $\mathbf{v}(\lambda)$ to have lower degree than this upper bound would require the simultaneous cancellation of all terms of highest degree in all components of the vector.

Definition 7.3 (Predictable Degree Property). A polynomial linear combination $\mathbf{v}(\lambda)$ of vector polynomials defined as in (7.19) has the predictable degree property (PDP) if

$$
\operatorname{deg} \mathbf{v}(\lambda)=\max _{j}\left(\operatorname{deg}\left(p_{j} \mathbf{v}_{j}\right)\right)=\max _{j}\left(\operatorname{deg} p_{j}+\operatorname{deg} \mathbf{v}_{j}\right)
$$

By extension, a vector polynomial basis $\mathcal{B}=\left\{\mathrm{v}_{j}(\lambda)\right\}_{j=1, k}$ for a rational subspace $V \subseteq \mathbb{F}(\lambda)^{n}$ is said to have the predictable degree property if every polynomial linear combination built from $\mathcal{B}$ has the PDP.

REMARK 7.4. The predictable degree property in Definition 7.3 is a special case of a more general predictable degree property for products of polynomial matrices, as described in [5].

Theorem 7.5 (Minimal Bases have the PDP). Any minimal basis $\mathcal{B}$ (in the filtration sense) for a rational subspace $V \subseteq \mathbb{F}(\lambda)^{n}$ has the $P D P$.

Proof. Once again we can induct on the degree parameter $d$ in the filtration $\mathcal{F}_{\mathrm{deg}}(V)$. To begin the induction (at $d=0$ ), consider an arbitrary vector polynomial $\mathbf{v}(\lambda) \in \mathcal{S}_{0}(V)$, expressible (by Theorem 7.2 ) as a polynomial linear combination $\mathbf{v}(\lambda)=\sum_{\mathbf{v}_{i} \in \mathcal{B}_{0}} p_{i}(\lambda) \mathbf{v}_{i}$ of the (all constant) basis vectors in $\mathcal{B}_{0} \subseteq \mathcal{B}$. Letting

$$
m:=\max _{\mathbf{v}_{i} \in \mathcal{B}_{0}}\left(\operatorname{deg}\left(p_{i} \mathbf{v}_{i}\right)\right)=\max _{\mathbf{v}_{i} \in \mathcal{B}_{0}}\left(\operatorname{deg}\left(p_{i}(\lambda)\right)\right.
$$

our goal is to show that $\operatorname{deg} \mathbf{v}(\lambda)=m$. But this follows easily by expanding each polynomial coefficient as $p_{i}(\lambda)=c_{m}^{(i)} \lambda^{m}+($ l.o.t. $)$, where "l.o.t." denotes lower order terms, so that $\mathbf{v}(\lambda)$ can now be expressed by

$$
\mathbf{v}(\lambda)=\lambda^{m}\left(\sum_{\mathbf{v}_{i} \in \mathcal{B}_{0}} c_{m}^{(i)} \mathbf{v}_{i}\right)+(\text { l.o.t. })
$$

Since the coefficient of $\lambda^{m}$ is a nontrivial linear combination of the linear independent vectors in $\mathcal{B}_{0}$, we see that $\operatorname{deg} \mathbf{v}(\lambda)$ is indeed equal to $m$. Thus the minimal basis $\mathcal{B}_{0}$ for the rational subspace $\mathcal{S}_{0}(V)$ has the PDP.

To complete the induction, we show that if the basis $\mathcal{B}_{d-1}$ for the subspace $\mathcal{S}_{d-1}(V)$ has the PDP, then the same is true for the basis $\mathcal{B}_{d}$ for $\mathcal{S}_{d}(V)$; the proof will proceed in the contrapositive form. That is, suppose there exists a nonzero vector polynomial $\mathbf{w}(\lambda) \in \mathcal{S}_{d}(V)$, that when expressed in terms of the basis $\mathcal{B}_{d}$ does not have the PDP. Then from $\mathbf{w}(\lambda)$ we show how to construct a nonzero vector polynomial $\mathbf{z}(\lambda)$ in $\mathcal{S}_{d-1}(V)$ that also does not have the PDP, thus establishing the desired contrapositive implication. 
So consider $\mathbf{w}(\lambda)=\sum_{\mathbf{v}_{i} \in \mathcal{B}_{d}} p_{i}(\lambda) \mathbf{v}_{i}(\lambda) \in \mathcal{S}_{d}(V)$, and as before let

$$
m:=\max _{\mathbf{v}_{i} \in \mathcal{B}_{d}}\left(\operatorname{deg}\left(p_{i}(\lambda) \mathbf{v}_{i}(\lambda)\right)\right) .
$$

Note that if $m<d$, then $\mathbf{w}(\lambda)$ is itself in $\mathcal{S}_{d-1}(V)$, so taking $\mathbf{z}(\lambda):=\mathbf{w}(\lambda)$ completes the argument. Thus from now on we assume that $d \leq m$; also we have $\operatorname{deg} \mathbf{w}(\lambda)<m$, since $\mathbf{w}(\lambda)$ is assumed to violate the PDP. The first step in the construction of the desired $\mathbf{z}(\lambda) \in \mathcal{S}_{d-1}(V)$ is to remove all the terms $p_{i}(\lambda) \mathbf{v}_{i}(\lambda)$ from $\mathbf{w}(\lambda)$ that have $\operatorname{deg} p_{i} \mathbf{v}_{i}<m$, to form the deleted sum $\mathbf{w}^{\prime}(\lambda)=\sum^{\prime} p_{j}(\lambda) \mathbf{v}_{j}(\lambda)$ in which every term $p_{j} \mathbf{v}_{j}$ has degree $m$. Observe that this deleted sum is a nontrivial polynomial linear combination because of (7.20), hence is a nonzero vector polynomial, and still has $\operatorname{deg} \mathbf{w}^{\prime}(\lambda)<m$. Next expand each remaining coefficient $p_{j}(\lambda)$ as $p_{j}(\lambda)=c_{j} \lambda^{d_{j}}+$ (l.o.t.), where $d_{j}+\operatorname{deg} \mathbf{v}_{j}(\lambda)=m$ and each $c_{j} \neq 0$, and delete all the lower order terms (l.o.t.) to form the vector polynomial $\widetilde{\mathbf{w}}^{\prime}(\lambda)=\sum^{\prime} c_{j} \lambda^{d_{j}} \mathbf{v}_{j}(\lambda)$. This $\widetilde{\mathbf{w}}^{\prime}(\lambda)$ is again a nonzero vector polynomial with each individual term $c_{j} \lambda^{d_{j}} \mathbf{v}_{j}(\lambda)$ having degree $m$, but $\operatorname{deg} \widetilde{\mathbf{w}}^{\prime}(\lambda)<m$; hence $\widetilde{\mathbf{w}}^{\prime}(\lambda)$ violates the PDP. The final step in the construction of $\mathbf{z}(\lambda)$ is to observe that each $d_{j} \geq m-d$, and so

$$
\mathbf{z}(\lambda):=\left(\frac{1}{m-d}\right) \widetilde{\mathbf{w}}^{\prime}(\lambda)=\sum^{\prime} c_{j} \lambda^{d_{j}-m+d} \mathbf{v}_{j}(\lambda),
$$

is a nonzero vector polynomial. Furthermore, each term $c_{j} \lambda^{d_{j}-m+d} \mathbf{v}_{j}(\lambda)$ in this summation defining $\mathbf{z}(\lambda)$ has degree $d$, but $\operatorname{deg} \mathbf{z}(\lambda)<d$. Thus $\mathbf{z}(\lambda)$ is a nonzero vector polynomial that does not have the PDP, but is an element of $\mathcal{P}_{d-1}(V)$, hence also of $\mathcal{S}_{d-1}(V)$. The existence of such a vector polynomial then completes the proof of the inductive step.

Theorems 7.2 and 7.5 have shown that any minimal basis has both the PDP and the PLC properties. But more than this is true. In fact these two properties characterize when a polynomial basis is minimal. That is, a vector polynomial basis $\mathcal{B}$ is minimal if and only if $\mathcal{B}$ has both the PDP and PLC properties. This result was shown in [7] using Forney's definition of minimal basis; in the final theorem of this paper we complete the argument for this characterization from the filtration point of view.

TheOREm 7.6 (Characterization of Minimal Bases). A vector polynomial basis $\mathcal{B}$ for a rational subspace $V \subseteq \mathbb{F}(\lambda)^{n}$ is a minimal basis (in the filtration sense) if and only if it has the PDP and PLC properties.

Proof. All that remains is to show that for a vector polynomial basis $\mathcal{B}$, the properties PDP and PLC together imply that $\mathcal{B}$ is a minimal basis. We consider this in the contrapositive form, i.e., we show that the non-minimality of a basis $\mathcal{B}$ implies that either PDP or PLC fails to hold.

So suppose $V \subseteq \mathbb{F}(\lambda)^{n}$ is a $k$-dimensional rational subspace with minimal indices $\varepsilon_{1} \leq \varepsilon_{2} \leq \cdots \leq \varepsilon_{k}$, and $\mathcal{B}=\left\{\mathbf{w}_{1}(\lambda), \mathbf{w}_{2}(\lambda), \ldots, \mathbf{w}_{k}(\lambda)\right\}$ is any vector polynomial basis for $V$, ordered so that the degrees $\delta_{i}=\operatorname{deg} \mathbf{w}_{i}(\lambda)$ satisfy $\delta_{1} \leq \delta_{2} \leq \cdots \leq \delta_{k}$. Then assuming that $\mathcal{B}$ is not minimal (in the filtration sense), we aim to show that either the PDP or the PLC property fails for $\mathcal{B}$. By the strong minimality property (Theorem 4.2) we know that $\varepsilon_{1} \leq \delta_{1}, \varepsilon_{2} \leq \delta_{2}, \ldots, \varepsilon_{k} \leq \delta_{k}$, and since $\mathcal{B}$ is not minimal, by Corollary 3.14 there must be at least one index where equality does not hold. Let $\ell$ be the first index where we have strict inequality; i.e., $\varepsilon_{1}=\delta_{1}, \varepsilon_{2}=\delta_{2}, \ldots, \varepsilon_{\ell-1}=\delta_{\ell-1}$, but $\varepsilon_{\ell}<\delta_{\ell}=\operatorname{deg} \mathbf{w}_{\ell}(\lambda)$. With $d:=\varepsilon_{\ell}$, it is clear that $\operatorname{dim} \mathcal{S}_{d}(V) \geq \ell$, so there must be some degree $d$ vector polynomial $\mathbf{v}(\lambda)$ in $\mathcal{S}_{d}(V) \backslash \operatorname{span}\left\{\mathbf{w}_{1}(\lambda), \mathbf{w}_{2}(\lambda), \ldots, \mathbf{w}_{\ell-1}(\lambda)\right\}$.

Now we claim that any such $\mathbf{v}(\lambda)$ is a witness to the failure of either the PDP or the PLC property for the basis $\mathcal{B}$. Certainly $\mathbf{v}(\lambda)$ can be (uniquely) expanded as a rational linear combination

$$
\mathbf{v}(\lambda)=\sum_{\mathbf{w}_{i} \in \mathcal{B}} r_{i}(\lambda) \mathbf{w}_{i}(\lambda)
$$


of the basis vectors in $\mathcal{B}$, with at least one nonzero coefficient $r_{j}(\lambda)$ with $\ell \leq j \leq k$. On the one hand, if any of the nonzero coefficients $r_{i}(\lambda)$ in (7.21) is not polynomial, then $\mathcal{B}$ would fail to have the PLC property. On the other hand, if the linear combination (7.21) does have all polynomial coefficients, then $\mathcal{B}$ would not have the PDP, since $d=\operatorname{deg} \mathbf{v}(\lambda)$ would be strictly less than the maximal degree $m$ of the terms $r_{j}(\lambda) \mathbf{w}_{j}(\lambda)$ in the summation (7.21); indeed, $m \geq \delta_{\ell}>\varepsilon_{\ell}=d$. Thus at least one of the properties (PDP or PLC) is violated by any non-minimal basis $\mathcal{B}$.

8. Conclusions. We have shown how the Kronecker/Gantmacher approach to the minimal bases and indices of any rational subspace $V \subseteq \mathbb{F}(\lambda)^{n}$ can be reformulated in a more intrinsic fashion using the degree filtration of $V$. This reformulation unifies and simplifies our understanding of the classical approaches to minimal bases and indices, clarifies the relationship between these various approaches, and provides new tools with which to prove basic properties of minimal indices and minimal bases. These new tools have been utilized to show that the minimal indices of any singular matrix polynomial are unchanged by field extension, and to prove the direct sum property of minimal indices. The filtration point of view has also provided deeper insight into minimal indices, bringing an under-recognized strong minimality property clearly into the light, thereby leading to a characterization of the vector polynomial bases in rational vector spaces. The efficacy and naturalness of this new approach have been further highlighted by finding new arguments for the previously known polynomial linear combination and predictable degree properties of minimal bases. Additional results and insights about minimal indices and bases can be expected to emerge from continued development of the filtration point of view.

Acknowledgements. I thank Froilán Dopico, Niloufer Mackey, Vasilije Perović, and Ion Zaballa for helpful comments on earlier versions of this paper. I also thank Paul Fuhrmann and Uwe Helmke for citing an earlier preprint [16] of this article in their recent book [8].

\section{REFERENCES}

[1] G. Ammar and C. Martin. The geometry of matrix eigenvalue methods. Acta Appl. Math., 5:239-278, 1986.

[2] I. Baragaña and I. Zaballa. Feedback invariants of supplementary pairs of matrices. Automatica, 33:2119-2130, 1997.

[3] F. De Terán and F. Dopico. Low rank perturbation of Kronecker structures without full rank. SIAM J. Matrix Anal. Appl., 29:496-529, 2007.

[4] F. De Terán, F. Dopico, and D.S. Mackey. Spectral equivalence of matrix polynomials and the index sum theorem. Linear Algebra Appl., 459:264-333, 2014.

[5] F. Dopico, D.S. Mackey, and P. Van Dooren. Product realizations of structural data for matrix polynomials. In preparation.

[6] G.D. Forney. Convolutional codes I: algebraic structure. IEEE Trans. Information Theory, IT-16:720-738, 1970.

[7] G.D. Forney. Minimal bases of rational vector spaces, with applications to multivariable linear systems. SIAM J. Control, 13:493-520, 1975.

[8] P. Fuhrmann and U. Helmke. The Mathematics of Networks of Linear Systems. Springer, Cham, 2015.

[9] F.R. Gantmacher. Theory of Matrices, Vols. I and II. Chelsea, New York, 1959.

[10] I. Gohberg and L. Lerer. Factorization indices and Kronecker indices of matrix polynomials. Integral Equ. Oper. Theory, $2: 199-243,1979$.

[11] P.J. Hilton and S. Wylie. Homology Theory: An Introduction to Algebraic Topology. Cambridge University Press, Cambridge, 1967.

[12] R.A. Horn and C.R. Johnson. Matrix Analysis. Cambridge University Press, Cambridge, 1985.

[13] J.H. Hubbard and B.H. West. Differential Equations: A Dynamical Systems Approach: Higher-Dimensional Systems. Springer-Verlag, New York, 1995.

[14] J. Jordan and U. Helmke. Controllability of the $Q R$-algorithm on Hessenberg flags, In: D. Gilliam and J. Rosenthal (editors), Proceedings of the Fifteenth International Symposium on Mathematical Theory of Networks and Systems (MTNS 2002), 2002. 
[15] T. Kailath. Linear Systems. Prentice Hall, Englewood Cliffs, NJ, 1980.

[16] D.S. Mackey. Minimal indices and minimal bases via filtrations. MIMS EPrint 2012.82. Manchester Institute for Mathematical Sciences, The University of Manchester, UK, 20 January 2014.

[17] R.J. McEliece. The algebraic theory of convolutional codes. In: V. Pless and W.C. Huffman (editors) Handbook of Coding Theory, Vol. 1, Elsevier, Amsterdam, 1065-1138, 1998.

[18] R.J. McEliece and R.P. Stanley. The general theory of convolutional codes. In E.C. Posner (editor), Telecommunications and Data Acquisition Progress Report 42-113, January-March 1993, NASA Jet Propulsion Laboratory, 89-98, 1993.

[19] H.H. Rosenbrock. Minimal indices in dynamical systems. Electron. Lett., 5(25):658-659, 1969.

[20] J. Rosenthal. Minimal bases of rational vector spaces and their importance in algebraic system theory. In: R.E. Blahut and R. Koetter (editos), Codes, Graphs, and Systems, Kluwer, Dordrecht, 345-357, 2002.

[21] H. Shapiro. The Weyr characteristic. Amer. Math. Monthly, 106:919-929, 1999.

[22] M. Shub and A.T. Vasquez. Some linearly induced Morse-Smale systems, the $Q R$-algorithm, and the Toda lattice. Contemporary Mathematics, Vol. 64, The Legacy of Sonya Kovalevskaya, AMS, Providence, 181-194, 1987.

[23] E.D. Sontag. Mathematical Control Theory: Deterministic Finite-Dimensional Systems, 2nd edition. Springer, New York, 1998.

[24] P. Van Dooren. The computation of Kronecker's canonical form of a singular pencil. Linear Algebra Appl., 27:103-140, 1979.

[25] I. Zaballa. Personal communication. June 4, 2013. 